\title{
Imaging Study of Pseudotumoral Chronic Sialadenitis with Cystic-Like Pattern in a Sjögren Syndrome Patient
}

\author{
Stefan-Cristian Dinescu ${ }^{1}$ (D), Anca Emanuela Musetescu ${ }^{1, *}$, Paulina Lucia Ciurea ${ }^{1}$, \\ Diana Monica Girnita ${ }^{2}$, Sineta Cristina Firulescu ${ }^{1}$ and Ana-Maria Bumbea ${ }^{3}$ \\ 1 Department of Rheumatology, Faculty of Medicine, University of Medicine and Pharmacy of Craiova, \\ Craiova 200349, Romania; stefandinescu@yahoo.com (S.-C.D.); ciureapaulina@yahoo.com (P.L.C.); \\ sineta.firulescu@gmail.com (S.C.F.) \\ 2 Faculty for Internal Medicine, Trihealth Good Samaritan Hospital, Cincinnati, OH 45220, USA; \\ dianagirnita@gmail.com \\ 3 Department of Medical Rehabilitation, Faculty of Medicine, University of Medicine and Pharmacy of \\ Craiova, Craiova 200349, Romania; anamariabumbea@yahoo.com \\ * Correspondence: anca_musetescu@yahoo.com; Tel.: +40-723-055-385
}

Received: 12 February 2018; Accepted: 6 March 2018; Published: 7 March 2018

\begin{abstract}
Ultrasonography has become a valuable tool for the assessment of salivary gland involvement in Sjögren syndrome. Diagnostic pitfalls can be encountered in late stages of the disease, in which morphologic changes may overlap with other pathologies of the salivary glands. We present the case of a female Sjögren syndrome patient with lack of significant sicca symptoms and unilateral occurrence of parotidomegaly, which prompted the suspicion of a parotid gland tumor. Due to the atypical clinical profile, diagnosis of Sjögren syndrome was delayed, at which point, severe sialadenitis produced a cystic transformation of the parotid gland parenchyma.
\end{abstract}

Keywords: ultrasonography; Sjögren's syndrome; parotid gland; sialadenitis

\section{Introduction}

The ultrasound (US) assessment of parotid glands (PG) has become a common practice in the diagnostic work-up of Sjögren syndrome (SS) patients. The hallmark US feature in SS is parenchymal inhomogeneity (PIH), a structural change that occurs as a result of local lymphocytic infiltrate [1]. Ongoing research has helped to establish different grading systems based on the PIH, and has also hinted at the potential inclusion of the US exam in the currently accepted diagnostic criteria [2]. Appropriate evaluation by a rheumatologist may be greatly delayed in patients who tolerate a casual xerostomia or lack sicca symptoms altogether. Diagnostic difficulties may be encountered if advanced structural changes have developed, and if imaging features mimic other PG pathologies, such as neoplastic or granulomatous disease [3]. We present the case of a female SS patient with scarce clinical symptoms, initially diagnosed with salivary gland tumor, in which the severe sialadenitis displayed atypical US features, with a predominantly cystic-like pattern.

\section{Case Report}

We report the case of a 58-year-old female patient, with no significant disease history, except for a slow growing mass on her left mandible, which became stiffer over time. The patient did not report any symptoms associated with impaired function of the salivary or lacrimal glands. She was referred to a surgery department and, after raising the suspicion of a PG tumor, a computed tomography (CT) scan was performed. On CT scans, both PGs displayed pronounced structural abnormalities with 
predominant cystic transformation and multiple calcifications (Figure 1a), while on the CT sialography marked sialectasis, of over $7 \mathrm{~mm}$ bilaterally, and no evidence of other potential obstruction in the ductal system were depicted (Figure 1b).

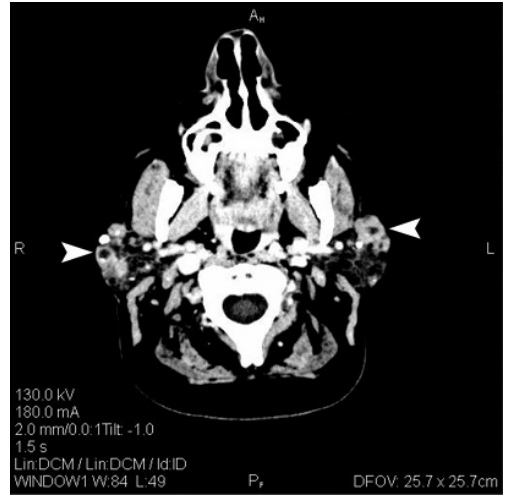

(a)

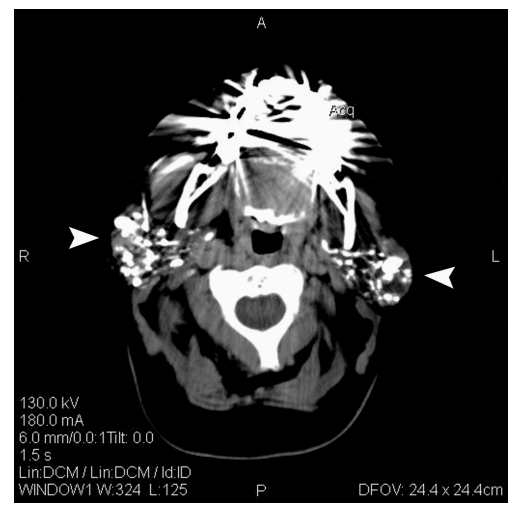

(b)

Figure 1. (a) CT (computed tomography) scan, axial section of the head, both PGs (parotid glands) are enlarged and display structural abnormalities, with multiple cystic formations (arrowheads); (b) CT sialography scan displays bilateral marked sialectasis with quasi-total involvement of glandular parenchyma (arrowheads).

At this point, she was admitted to our department in order to further evaluate a possible autoimmune etiology. Serologic profile revealed positive anti-Ro-52 and anti-SS-A antibodies, negative anti-La/SSB antibodies and rheumatoid factor. Schirmer test was negative. Histopathology exam confirmed the presence of lymphocytic aggregates (Figure 2a), with predominantly CD20+ B cell subpopulation on immunohistochemistry exam, some with pseudonodular distribution, associated with marked sialectasis (Figure 2b).

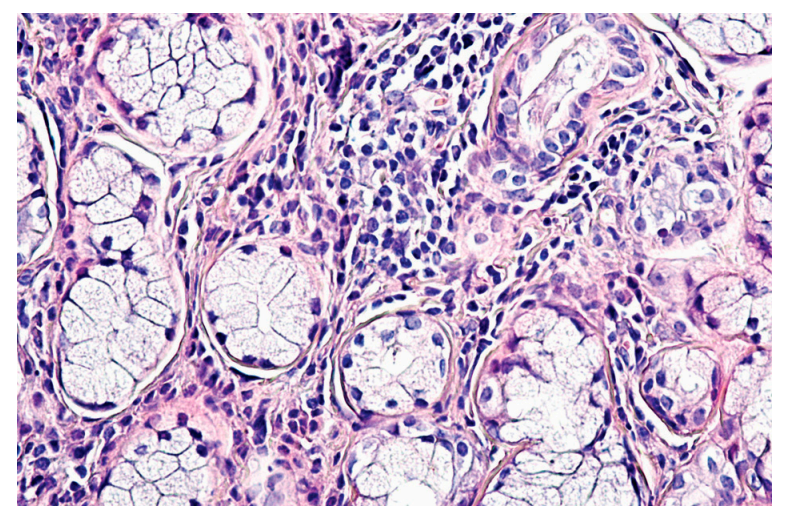

(a)

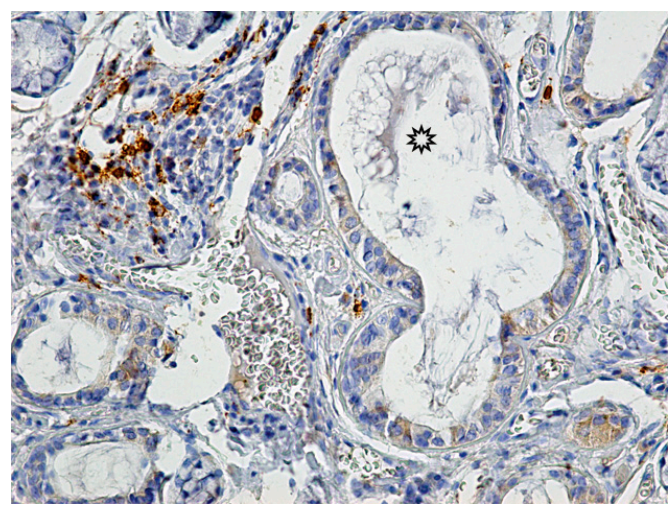

(b)

Figure 2. (a) minor salivary gland histopathology features on Hematoxylin-Eosin stain show lymphocytic sialadenitis as mononuclear cell aggregates adjacent to glandular acini; (b) CD20 immunostaining, 200×, distribution of B cell subpopulation and marked sialectasis (asterisk).

On grey-scale US images, the structure of the glandular parenchyma was greatly altered. Both PGs displayed intense hypo/anechoic areas, some exceeding $6 \mathrm{~mm}$ in diameter (Figure 3a). These structural changes were distributed to a significant extent throughout both PGs, having a near total involvement. Homogenous areas of residual glandular tissue were scarcely observed. Based on the Salaffi et al. scoring system [4], a grade 4 PIH was established. In Doppler mode, increased vascularity was observed in the residual tissue (Figure $3 b$ ), scoring 2 on a semi-quantitative scale [5]. 


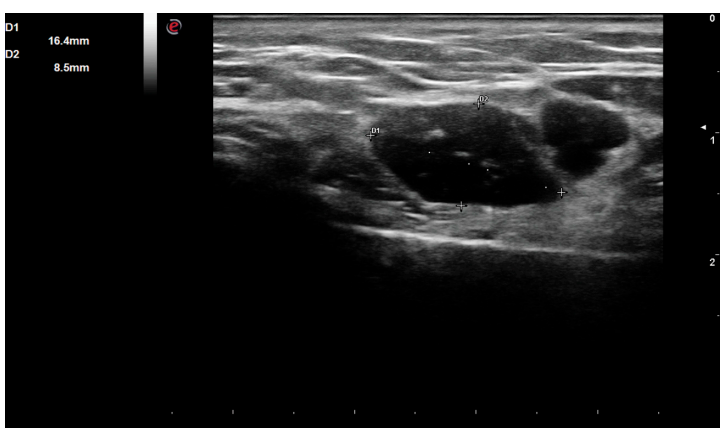

(a)

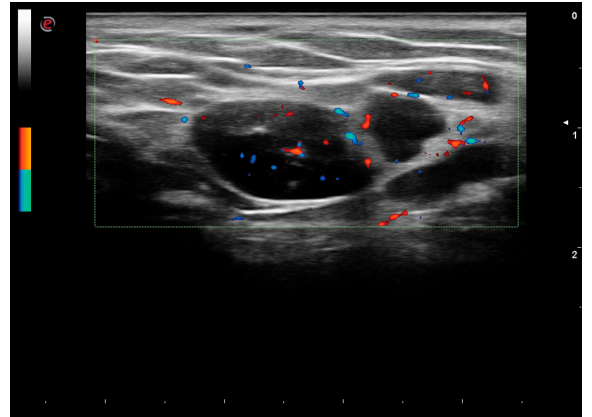

(b)

Figure 3. US (ultrasound) image of PGs, longitudinal section. (a) left PG displays severe structural changes, multiple large and well-defined, almost anechoic areas, with a maximum diameter of $16 \mathrm{~mm}$; (b) power Doppler of left PG shows a moderate vascularization index, with spots inside the hypoechoic areas and along the residual glandular tissue surrounding the cysts.

Through the relative adjacent distribution of hypoechoic areas, well-defined margins and surrounding tissue resembling septa, the PGs acquired a more cystic-like pattern, with limited functional tissue (Figure 4a). Multiple hyperechoic formations, with posterior acoustic echo, pointed to the development of sialolithiasis (Figure $4 \mathrm{~b}$ ). We also examined the degree of tissue stiffness using the Acoustic Radiation Force Impulse (ARFI) technique (Figure 4c). We performed 5 measurements on each PG and obtained a mean overall shear wave velocity of $2.06 \mathrm{~m} / \mathrm{s}$. This measurement could not be interpreted as pathologic, due to the limited data published so far concerning reference values in healthy subjects [6], and due to a lack of established cut-off values. A written informed consent was obtained from the patient regarding the inclusion in a research project and eventual publishing of medical data.

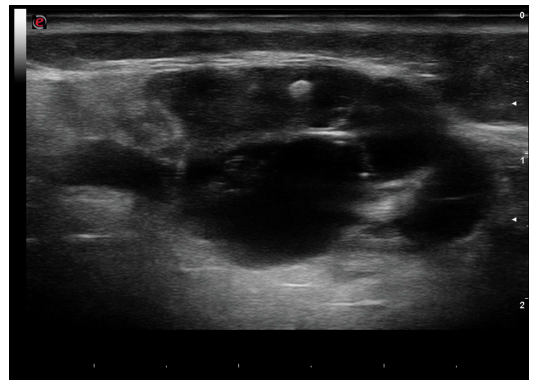

(a)

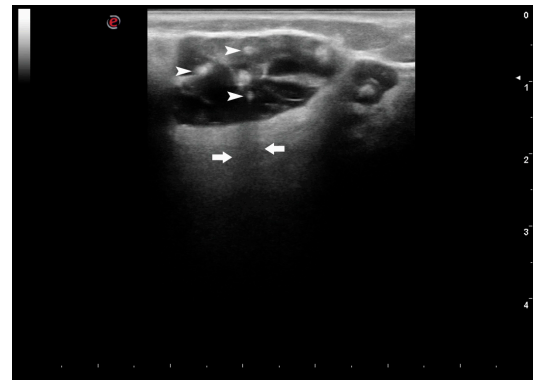

(b)

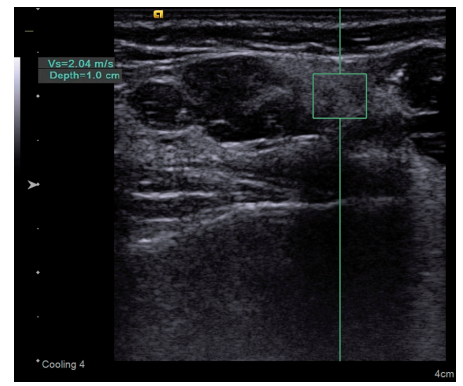

(c)

Figure 4. (a) PG parenchyma displaying pseudotumoral features, with cystic-like pattern; (b) sialolithiasis shown as multiple hyperechoic formations (arrowheads) with posterior acoustic attenuation (arrows); (c) ARFI US of PG, with region of interest set at $1 \mathrm{~cm}$ under the skin, quantifies the grade of glandular stiffness at $2.04 \mathrm{~m} / \mathrm{s}$.

\section{Discussion}

The main pathologic feature that develops in SS is autoimmune destructive sialadenitis, with focal mononuclear cell aggregates, predominantly in the periductal areas [7] and subsequent development of sicca symptoms. Particularities encountered in our patient reside in the lack of significant sicca symptoms and also the unilateral occurrence of parotidomegaly, prompting the suspicion of a PG tumor. Curiously, the functional reserve of PGs was provided by the scarce residual tissue, which maintained sufficient secretory function.

The main US features observed in SS include decreased parenchymal echogenity, multiple small, oval hypoechoic areas, unclear borders and hyperechogenic reflections. All these changes account 
for an overall PIH of the salivary glands [1]. Further grading of the PIH is established through semi-quantitative scoring systems $[4,8,9]$. Although studies on US features of salivary glands in SS have helped outline some common features, they remain mostly unspecific, especially when the clinical profile does not support a clear diagnosis. In the presence of multiple hypoechoic areas scattered in the salivary gland parenchyma, one must also take in consideration other types of chronic sialadenitis, such as Küttner tumor and granulomatous disease (e.g., sarcoidosis), HIV parotiditis or hematogenous metastases [3]. Structural damage to the salivary glands with similar clinical and imaging features to SS can also develop in an underlying hepatitis $\mathrm{C}$ virus (HCV) infection, in which sialotropism of $\mathrm{HCV}$ can lead to chronic sialdenitis [10]. In advanced stages of SS, severe ductal inflammation causes marked intraglandular sialectasis, which in turn may result in the formation of numerous cystic spaces. At this point, US exam reveals large anechoic areas with well-defined margins [11].

In a study by Takashima S. et al., PG pseudotumors were divided into solid types, formed by local massive lymphocytic infiltration, and cystic types produced by severely dilated intralobular ducts [12]. Benign tumors of the PG, such as pleomorphic adenoma and Warthin tumor, can display cystic features, but are usually solitary and unilateral. Conditions associated with bilateral cystic structures include acquired immunodeficiency syndrome, polycystic disease of the PG, pseudocystic appearance of lymphoma or metastatic lymph nodes with central fluid collection [3].

\section{Conclusions}

Atypical clinical presentation of SS may considerably delay a correct diagnosis. Although US has become a valuable tool for the assessment of salivary gland involvement in SS, diagnostic pitfalls can be encountered in late stages of the disease, in which morphologic features may overlap with other pathologies of the salivary glands.

Author Contributions: Stefan-Cristian Dinescu and Anca Emanuela Musetescu collected the patient data and performed the imaging study; Sineta Cristina Firulescu and Ana-Maria Bumbea contributed with the literature review and wrote the paper; Paulina Lucia Ciurea and Diana Monica Girnita contributed to the proof reading and final editing.

Conflicts of Interest: The authors declare no conflict of interest.

\section{Abbreviations}

$\begin{array}{ll}\text { US } & \text { Ultrasound } \\ \text { PG } & \text { Parotid Glands } \\ \text { SS } & \text { Sjögren Syndrome } \\ \text { PIH } & \text { Parenchymal Inhomogeneity } \\ \text { CT } & \text { Computed Tomography } \\ \text { ARFI } & \text { Acoustic Radiation Force Impulse } \\ \text { HCV } & \text { Hepatitis C Virus }\end{array}$

\section{References}

1. Jousse-Joulin, S.; Milic, V.; Jonsson, M.V.; Plagou, A.; Theander, E.; Luciano, N.; Rachele, P.; Baldini, C.; Bootsma, H.; Vissink, A.; et al. Is salivary gland ultrasonography a useful tool in Sjogren's syndrome? A systematic review. Rheumatology 2016, 55, 789-800. [CrossRef] [PubMed]

2. Vitali, C.; Bombardieri, S.; Jonsson, R.; Moutsopoulos, H.M.; Alexander, E.L.; Carsons, S.E.; Daniels, T.E.; Fox, P.C.; Fox, R.I.; Kassan, S.S.; et al. Classification criteria for Sjögren's syndrome: A revised version of the European criteria proposed by the American-European Consensus Group. Ann. Rheum. Dis. 2002, 61, 554-558. [CrossRef] [PubMed]

3. Bialek, E.J.; Jakubowski, W.; Zajkowski, P.; Szopinski, K.T.; Osmolski, A. US of the major salivary glands: Anatomy and spatial relationships, pathologic conditions, and pitfalls. Radiographics 2006, 26, 745-763. [CrossRef] [PubMed] 
4. Salaffi, F.; Argalia, G.; Carotti, M.; Giannini, F.; Palombi, C. Salivary gland ultrasonography in the evaluation of primary Sjogren's syndrome. Comparison with minor salivary gland biopsy. J. Rheumatol. 2000, 27, 1229-1236. [PubMed]

5. Martinoli, C.; Derchi, L.E.; Solbiati, L.; Rizzatto, G.; Silvestri, E.; Giannoni, M. Color Doppler sonography of salivary glands. Am. J. Roentgenol. 1994, 163, 933-941. [CrossRef] [PubMed]

6. Samier-Guérin, A.; Saraux, A.; Gestin, S.; Cornec, D.; Marhadour, T.; Devauchelle-Pensec, V.; Bressollette, L.; Nonent, M.; Jousse-Joulin, S. Can ARFI elastometry of the salivary glands contribute to the diagnosis of Sjögren's syndrome? Jt. Bone Spine Rev. Rhum. 2015, 83, 301-306. [CrossRef] [PubMed]

7. Dinescu, S.C.; Fortofoiu, M.C.; Bumbea, A.M.; Ciurea, P.L.; Busuioc, C.J.; Muşetescu, A.E. Histopathological and immunohistochemical profile in primary Sjogren s syndrome. Rom. J. Morphol. Embryol. 2017, 58, 409-417. [PubMed]

8. Hocevar, A.; Ambrozic, A.; Rozman, B.; Kveder, T.; Tomsic, M. Ultrasonographic changes of major salivary glands in primary Sjogren's syndrome. Diagnostic value of a novel scoring system. Rheumatology 2005, 44, 768-772. [CrossRef] [PubMed]

9. Carotti, M.; Salaffi, F.; Manganelli, P.; Argalia, G. Ultrasonography and colour doppler sonography of salivary glands in primary Sjogren's syndrome. Clin. Rheumatol. 2001, 20, 213-219. [CrossRef] [PubMed]

10. Dinescu, S.C.; Ciurea, P.; Vreju, F.; Săndulescu, D.L.; Musetescu, A.E. Hepatitis C virus induced Sjogren syndrome: clinical and imaging features. Curr. Health Sci. J. 2017, 43, 78-82.

11. Tzioufas, A.G.; Moutsopoulos, H.M. Ultrasonography of salivary glands: an evolving approach for the diagnosis of Sjogren's syndrome. Nat. Clin. Pract. Rheumatol. 2008, 4, 454-455. [CrossRef] [PubMed]

12. Takashima, S.; Nagareda, T.; Noguchi, Y.; Takeuchi, N.; Tomiyama, N.; Johkoh, T.; Ikezoe, J.; Kozuka, T. CT and MR appearances of parotid pseudotumors in Sjogren syndrome. J. Comput. Assist. Tomogr. 1992, 16, 376-383. [CrossRef] [PubMed]

(C) 2018 by the authors. Licensee MDPI, Basel, Switzerland. This article is an open access article distributed under the terms and conditions of the Creative Commons Attribution (CC BY) license (http:/ / creativecommons.org/licenses/by/4.0/). 